\title{
Elektronische Abstimmungen und Wahlen
}

Bei elektronischen Abstimmungen und Wahlen gibt es eine Vielzahl von Lösungen, wobei ortsund zeitunabhängige Optionen via Internet im Vordergrund stehen. Im Beitrag werden die Teilprozesse für Abstimmungen (eVoting) und Wahlen (eElection) im Detail erläutert. Die Identifikation und Authentifikation des Wählers erfolgt in der Phase der Registrierung, in einer zweiten Phase muss die elektronische Stimmabgabe für Abstimmungen und Wahlen anonym erfolgen. Danach gelangen mehrdimensionale Visualisierungstechniken zur Darstellung der Abstimmungs- und Wahlergebnisse zur Anwendung (ePosting). Das politische und kulturelle Gedächtnis (Public Memory), im eGovernment auf Portalen und mit digitalen Archiven realisiert, erweitert die Optionen einer Informations- und Wissensgesellschaft und lässt ein demokratisches Politcontrolling zu.

\section{Inhaltsübersicht}

1 Zur Vielfalt elektronischer Abstimmungen und Wahlen

2 Prozessschritte für eVoting und eElection

3 Funktionsweise anonymer Abstimmungen und Wahlen

4 Analyse und Visualisierung mehrdimensionaler Wahlergebnisse

5 Schritte zum Public Memory

6 Literatur

\section{Zur Vielfalt elektronischer Abstimmungen und Wahlen}

Die Nutzung von Informations- und Kommunikationssystemen erlaubt den Bürgerinnen und Bürgern, ihre politischen Rechte (Stimmabgabe, Wahl, Unterschriften bei Referenden und Initiativen etc.) elektronisch wahrzunehmen [Meier
2002; Nurmi et al. 1991]. Die Tabelle 1 zeigt die Vielfalt bei elektronischen Abstimmungen und Wahlen.

Unter einem Web Poll versteht man eine webbasierte Umfrage zu einem öffentlichen Sachgeschäft oder einem politischen Programm, wobei das elektronisch ermittelte Resultat als unverbindlich taxiert werden muss. Mit anderen Worten werden die Bürgerinnen und Bürger nicht in jedem Fall eindeutig identifiziert und mit digitalen Signaturen auf ihre Authentifikation hin überprüft, vielmehr soll ein Stimmungsbild zu einem öffentlichen Sachgeschäft oder Thema eingefangen werden.

Elektronische Abstimmungssysteme können an Versammlungsorten oder in Sälen installiert werden, damit jeder Teilnehmer über ein elektronisches Abstimmungs- oder Wahlgerät verfügt. Die einzelnen Stimmen werden nach der Stimmabgabe elektronisch zusammengezählt und auf Anzeigetafeln direkt veröffentlicht. Solche Abstimmungssysteme sind meistens in Parlamentssälen fest installiert, zudem lassen sie sich mit begrenztem Aufwand bei öffentlichen Versammlungen ad hoc aufbauen und nutzen.

Als weitere Option bei elektronischen Abstimmungen und Wahlen können sogenannte Wahlcomputer oder elektronische Wahlmaschinen in den Wahllokalen aufgestellt und je nach Bedarf miteinander vernetzt werden. Bei den Wahlcomputern ist es möglich, bestehende PCs oder Arbeitsplatzrechner mit einer speziellen Abstimmungs- und Wahlsoftware zu versehen. Bei elektronischen Wahlmaschinen gelangen Spezialgeräte zum Einsatz, die ausschließlich für elektronische Abstimmungen und Wahlen konzipiert sind. Damit soll verhindert werden, dass die Abgabe von Stimmen 


\begin{tabular}{|c|c|c|c|}
\hline & Definition & Merkmale & Beispiele \\
\hline Web Polls & $\begin{array}{l}\text { unverbindliche } \\
\text { Abstimmung oder Wahl }\end{array}$ & $\begin{array}{l}\text { - keine Garantie auf } \\
\text { Korrektheit } \\
\text { - keine Garantie auf } \\
\text { Anonymität }\end{array}$ & $\begin{array}{l}\text { unverbindliche } \\
\text { Bürgerbefragung }\end{array}$ \\
\hline $\begin{array}{l}\text { elektronische } \\
\text { Abstimmungssysteme }\end{array}$ & $\begin{array}{l}\text { elektronische } \\
\text { Abstimmungsgeräte in } \\
\text { einem Saal }\end{array}$ & $\begin{array}{l}\text { - } \text { automatische Erfassung } \\
\text { - unmittelbare } \\
\text { Ergebnisanzeige }\end{array}$ & $\begin{array}{l}\text { Abstimmung im } \\
\text { Parlament }\end{array}$ \\
\hline $\begin{array}{l}\text { elektronische } \\
\text { Wahlmaschinen }\end{array}$ & $\begin{array}{l}\text { elektronische } \\
\text { Abstimmungsgeräte und } \\
\text { Urnen im Wahllokal }\end{array}$ & $\begin{array}{l}\text { - Stimmberechtigung der } \\
\text { Wähler manuell prüfen } \\
\text { - Ergebnisse einzelner } \\
\text { Maschinen manuell } \\
\text { zusammenfassen }\end{array}$ & Wahlen in Belgien \\
\hline $\begin{array}{l}\text { vernetzte } \\
\text { Wahlmaschinen }\end{array}$ & $\begin{array}{l}\text { vernetzte } \\
\text { Abstimmungsgeräte in } \\
\text { öffentlichen Wahllokalen }\end{array}$ & $\begin{array}{l}\text { - Ergebnisseautomatisch } \\
\text { für alle angeschlos- } \\
\text { senen Maschinen } \\
\text { ermittelbar }\end{array}$ & Lokalwahlen in England \\
\hline eVoting und eElection & $\begin{array}{l}\text { orts- und } \\
\text { zeitunabhängige } \\
\text { Abstimmungen u. } \\
\text { Wahlen durch Geräte } \\
\text { (Handy, Palmtop, } \\
\text { digitales TV u. a.) }\end{array}$ & $\begin{array}{l}\text { - Registrierung eindeutig } \\
\text { - Abstimmung oder Wahl } \\
\text { bleibt geheim }\end{array}$ & $\begin{array}{l}\text { Abstimmungen in Genf } \\
\text { und Zürich }\end{array}$ \\
\hline
\end{tabular}

Tab. 1: Unterschiede bei elektronischen Abstimmungen und Wahlen

und das Auslesen der Resultate manipuliert ${ }^{1}$ werden können.

Internetbasierte Abstimmungen (eVoting) und Wahlen (eElection) lassen sich in einem definierten Zeitfenster orts- und zeitunabhängig durchführen. Dabei erfolgen die Wähleridentifikation und die Wählerauthentifikation mit der Hilfe einer Public Key Infrastructure oder durch geeignete Sicherheitssysteme (siehe

1. In Deutschland z. B. gelangt der Wahlcomputer des Herstellers NEDAP in Gemeinden zum Einsatz. Durch Untersuchungen zur Sicherheit und Manipulierbarkeit konnte belegt werden (siehe [Kurz \& Rieger 2007]), mit welchen Methoden manipulierte Software in den Wahlcomputer eingebracht wird und wie Hardwarekomponenten ausgetauscht werden. Dieses Gerät erfüllt damit nicht die Anforderungen an manipulationsfeste, nachvollziehbare und transparente Abstimmungen und Wahlen.
Abschnitt 3), die eine Manipulation der Abstimmungs- und Wahlresultate ausschließen.

Zur Einführung eines elektronischen Wahlund Abstimmungssystems müssen die notwendigen gesetzlichen Grundlagen geschaffen werden. Die meisten europäischen Länder haben Erfahrungen mit unterschiedlichen elektronischen Abstimmungs- und Wahlverfahren gesammelt. Als Chancen werden die folgenden aufgeführt:

Umfassende Dokumentation vor, während und nach elektronischen Abstimmungen und Wahlen: Auf dem eGovernment-Portal können differenzierte und beliebig detaillierte Informationen, Persönlichkeitsprofile (vgl. Spinnenprofile politischer Mandatsträger in Abschnitt 4) und Entscheidungshilfen seitens der Behörden aufgeschaltet werden. Zudem lassen sich für Sachthemen und Wahlen entsprechende Links zu den politischen Parteien oder wichtigen 
NGOs (Nichtregierungsorganisationen) und NPOs (Non-Profit-Organisationen) hochschalten. Der Bürger entscheidet selber, wie tief er sich mit einem Thema oder einem Wahlvorschlag auseinandersetzen will. Zudem hat er die Möglichkeit, mit einem Abonnementsdienst (z. B. RSS-Feed) zur Informationsphase, Abstimmung, Wahl oder Analyse der Resultate auf dem Laufenden zu bleiben.

Erleichterung der Stimmabgabe: Die Mobilität der Bevölkerung nimmt zu, und es besteht ein Bedarf zur Ausübung politischer Rechte mit eVoting und eElection. Insbesondere profitieren behinderte Personen von diesen Möglichkeiten, wird ihnen doch der teilweise beschwerliche Gang zum Stimm- und Wahllokal erspart. Zudem können Bürgerinnen und Bürger, die sich außerhalb des Landes aufhalten, auf einfachere Art und Weise ihre Rechte ausüben.

Aktivierung der Bürgerinnen und Bürger: Elektronische Abstimmungen und Wahlen können dazu genutzt werden, Zusatzfragen zu den behördlichen Aufgaben und Pflichten zu stellen. Damit können nicht nur treffendere Aussagen zum laufenden Abstimmungsthema oder zu einer Wahl gemacht, sondern auch die ePartizipation der Bürgerinnen und Bürger angekurbelt werden.

Wie bei vielen Vorhaben im eGovernment bergen elektronische Abstimmungen und Wahlen neben den Chancen auch Risiken:

Entritualisierung des Abstimmungs- und Wahlvorgangs: Konventionelle Abstimmungen und Wahlen sind an Rituale und bestimmte Orte gebunden. Das Ausüben politischer Rechte mit internetbasierten Verfahren verlangt Verhaltensänderungen und ein Vertrauen in den Cyberspace. Ortsgebundene und föderalistische Strukturen müssen mit webbasierten Methoden und Techniken unterstützt und ermöglicht werden.

Flut von Web Polls und Entwertung der Volksrechte: Das Sammeln von Unterschriften für Initiativen oder Referenden wird durch webbasierte Verfahren erleichtert. Die Gefahr be- steht, dass öffentliche Themen und Projekte nicht differenziert ausdiskutiert werden, sondern dass spontan per Klick abgestimmt und beurteilt wird.

Datenschutz- und Datensicherheitsfragen: Obwohl konventionelle Abstimmungs- und Wahlverfahren ebenfalls Sicherheitsrisiken aufweisen, sind die elektronischen Verfahren bei vielen Bürgerinnen und Bürgern zurzeit noch wenig vertrauenserweckend. Mit abstrakten Verschlüsselungsmethoden und digitalen Signaturen sind viele Bürgerinnen und Bürger überfordert. Sie bleiben misstrauisch und zweifeln am Schutz ihrer Persönlichkeitssphäre. Der Missbrauch im Internet (rassistisches Gedankengut, Pädophilie, Kriminalität u.a.) schreckt davon ab, Bürgerrechte und Pflichten via Internet wahrzunehmen.

Das eGovernment-Framework der Universität Fribourg [Meier 2009] mit den Stufen Information und Kommunikation, Produktion und Partizipation suggeriert, dass die oberste Ebene der Partizipation mit eCollaboration, eDemocracy und eCommunity eine der schwierigsten Herausforderungen bleibt. Aus diesem Grunde ist es wichtig, erfolgreiche Teilprojekte mit eVoting und eElection durchzuführen, um die Schwellenängste der Bürgerinnen und Bürger abzubauen.

\section{Prozessschritte für eVoting und eElection}

Elektronische Abstimmungen und Wahlen [Brandt \& Volkert 2002] unterscheiden sich vor allem in den Vor- und Nachbearbeitungsphasen gegenüber herkömmlichen Abstimmungs- und Wahlverfahren (vgl. Abb. 1), falls die Vorteile elektronischer Austauschbeziehungen ausgeschöpft werden. Durch eine veränderte und erweiterte Informations- und Diskussionspolitik bei den Prozessschritten eDiscussion und ePosting erhofft man sich, Bürgerinnen und Bürger vermehrt für politische Anliegen zu gewinnen und die Community-Bildung zu fördern. 
In Abbildung 1 werden die folgenden Prozessschritte unterschieden:

eDiscussion: Im Vorfeld von Abstimmungen und Wahlen kann der Meinungsbildungsprozess gefördert werden, indem neben Informationen in Diskussionsforen Einschätzungen und Bewertungen eingeholt werden. Abonnementsdienste erlauben den Bürgerinnen und Bürgern, gewünschte Dokumente oder Entscheidungsgrundlagen nachzufragen sowie Änderungen und Erweiterungen bei Sachthemen zu erfahren.

eVoting: In der von der Behörde festgelegten Abstimmungszeit kann der Bürger seinen elektronischen Stimmzettel ausfüllen und abschicken [Krimmer 2006]. Vorher muss er sich bei der Behörde identifiziert und registriert haben, die Abstimmung erfolgt danach anonym (vgl. die zwei Phasen der Registrierung und der Abstimmung in Abschnitt 3). Die Behörde kann bei Bedarf neben dem Stimmzettel noch eine fakultative Erhebung über die vorliegenden Themen beifügen, um z. B. für Vorgehens- und Umsetzungsfragen ein Feedback von den Bürgerinnen und Bürgern zu erhalten.
eElection: Durch Publikation der Spinnenprofile der zu wählenden Mandatsträger (Abschnitt 4) und durch Zusatzinformationen zu den Fähigkeiten und Kenntnissen der Kandidatinnen und Kandidaten fällt es dem Wählenden im vorgelagerten Prozessschritt eDiscussion einfacher, den elektronischen Wahlzettel auszufüllen. Auch hier muss vorgängig mit einer Prüfkarte die Registrierung vorgenommen und von der Behörde ein gültiger Wahlzettel angefordert werden, bevor elektronisch abgestimmt werden kann (Abschnitt 3). Eventuell bietet es sich an, fakultative Zusatzfragen durch die Bürgerinnen und Bürger beantworten zu lassen.

ePosting: Eine Publikation der Resultate auf dem eGovernment-Portal der Behörde ist nicht nur für die Bürgerinnen und Bürger gedacht, sondern kann von anderen Organisationen oder der Presse studiert und weiterverwertet werden. Dazu können geeignete Visualisierungsund Auswertungswerkzeuge angeboten werden, sodass das Abstimmungs- oder Wahlverhalten sowie die Resultate analysiert und besprochen werden können. Public Blogs ermögli-

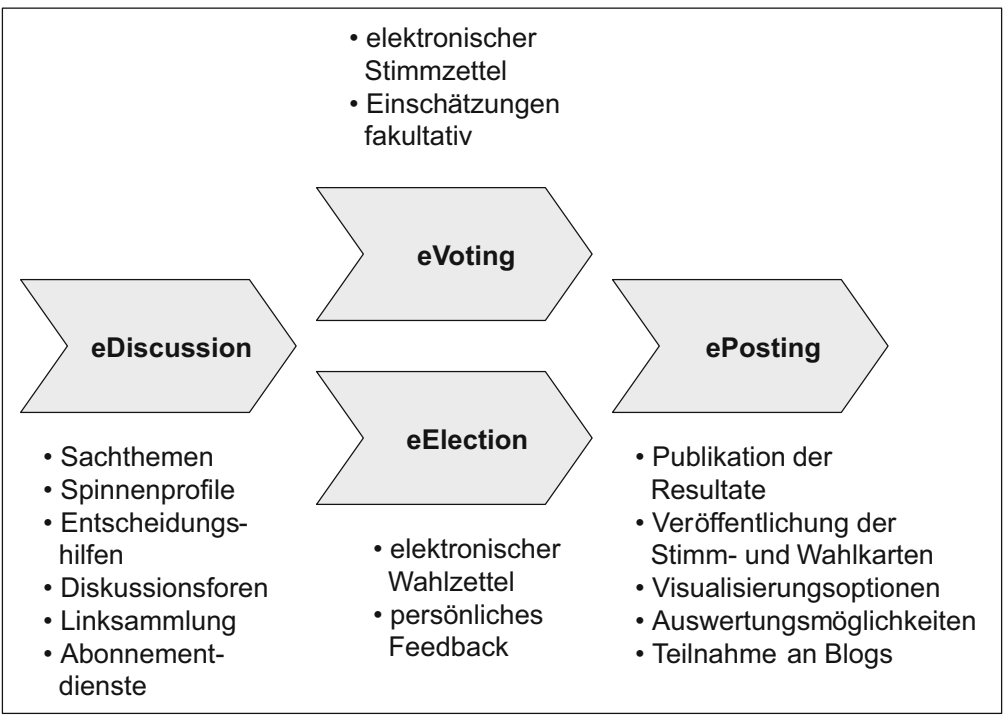

Abb. 1: eVoting und eElection als Teil einer Prozesskette 
chen es, die elektronische Abstimmung oder Wahl über den Stimm- und Wahltag hinaus zu kommentieren und zu vertiefen. Neben der Publikation der eigentlichen Abstimmungsoder Wahlresultate wäre es notwendig, Stimmund Wahlkarten bzw. deren eindeutige Identifikationsnummern in Listen zu veröffentlichen. Damit hat jeder Bürger die Möglichkeit, zu verifizieren, ob seine Stimme tatsächlich registriert und verarbeitet worden ist. Mit solcher gegenüber konventionellen Abstimmungen und Wahlen erweiterten Transparenz lässt sich das Vertrauen der Bürgerinnen und Bürger für eVoting und eElection steigern.

Die Beschreibung der Prozessschritte eDiscussion, eVoting, eElection und ePosting zeigen auf, dass bei der Nutzung elektronischer Informations- und Austauschbeziehungen das Involvement der Bürger gesteigert und die öffentliche Diskussion angeregt werden kann. Der Beitrag illustriert dazu in Abschnitt 3 das Vorgehen bei anonymen Abstimmungen und Wahlen; Methoden für den Prozessschritt ePosting werden in Abschnitt 4 mit Analyseund Visualisierungstechniken gegeben.

\section{Funktionsweise anonymer Abstimmungen und Wahlen}

Für die Durchführung von Abstimmungen via Internet müssen die bekannten Abstimmungsund Wahlrechtsgrundsätze gelten:

- Nur stimmberechtigte Personen können an elektronischen Abstimmungen und Wahlen teilnehmen.

- Jede stimmberechtigte Person hat eine und nur eine Stimme.

- Elektronisch abgegebene Stimmen dürfen weder abgefangen, umgeleitet noch verändert werden.

- Vom Inhalt elektronisch abgegebener Stimmen dürfen Dritte (Bürger, Behörde) keine Kenntnis erlangen (geheime Abstimmung oder Wahl).

- Der Datenschutz muss gewährleistet sein.
- Im Falle einer Panne darf keine bereits abgegebene elektronische Stimme verloren gehen.

Die Kernfrage bei elektronischen Abstimmungen und Wahlen lautet: Wie kann ein Bürger sich in einem elektronischen Austauschprozess eindeutig identifizieren, authentifizieren und für die Abstimmung oder Wahl autorisiert werden (vgl. Identity Management), wenn er danach eine anonyme bzw. geheime Stimme abgeben will? Von der rechtlichen wie technischen Seite her lehnt man sich beim eVoting wie bei der eElection an die in Europa (Deutschland, Frankreich und Schweiz) bewährte Briefwahl an. In Analogie dazu müssen die folgenden zwei Phasen [Prosser et al. 2002] beim elektronischen Stimm- und Wahlverfahren unterschieden werden:

Phase I - Registrierung: Der Wähler muss sich zunächst identifizieren und authentifizieren, um für die elektronische Abstimmung autorisiert zu werden.

Phase II - Stimmabgabe: In einem zweiten Schritt erfolgt die Stimmabgabe anonym, sodass kein Dritter Aufschluss über das Stimmoder Wahlverhalten des Wählers bekommen kann.

Für elektronische Abstimmungen und Wahlen ist eine Vielzahl von Systemen und Verfahren entwickelt worden. Bei elektronischen Wahlmaschinen lässt der Bürger seine Identität beim Wahlhelfer überprüfen und erhält eine Chipkarte, mit der er in der Wahlzelle seine Stimme elektronisch abgeben kann. Solche Systeme gelangen in den USA zum Einsatz ${ }^{2}$, sind aber für entfernte Abstimmungen und Wahlen weniger geeignet.

2. Nach den erheblichen Fehlerraten bei der Präsidentschaftswahl der USA in Florida im Jahr 2000 wurden mehrheitlich die mechanischen Wahlmaschinen durch elektronische ersetzt, um die aufgetretenen Unregelmäßigkeiten zu umgehen und Kontrollnachzählungen abzusichern. 
Bei der Nutzung von PIN-Codes (Personal Identification Number) oder ähnlich gelagerten Verfahren ist man bei der Forderung nach Anonymität von der Integrität der Wahlorganisatoren abhängig. Zudem entbehren diese Systeme meistens einer sauberen gesetzlichen Grundlage, wie sie beim Signaturgesetz für digitale Signaturen und der Public Key Infrastructure mit den Trustcentern gewährleistet ist. Im Folgenden soll deshalb ein Registrierungs- und Abstimmungsverfahren für eVoting und eElection skizziert werden, das den obigen Anforderungen korrekter und anonymer $\mathrm{Ab}$ stimmungen nachkommt.

In Abbildung 2 sind die beiden Phasen der Registrierung und der Stimmabgabe unabhängig voneinander konzipiert worden, um Anonymität zu garantieren [Prosser \& Müller-Török 2002].

In der Registrierungsphase wird der Wähler identifiziert und authentifiziert, indem er seine Wahlkarte verschlüsselt und signiert an die Registrierungsstelle schickt (Schritt 1). Der Registrierungsserver überprüft die Wahlberech- tigung des Wählers aufgrund seiner digitalen Signatur. Im positiven Fall retourniert der Server die Wahlkarte, und zwar blind 3 unterschrieben (Schritt 2). Der Wähler schickt im Schritt 3 die Wahlkarte (zur Unterscheidung wird sie jetzt als Prüfkarte gekennzeichnet) an ein Trustcenter und verlangt diese blind unterschrieben zurück (Schritt 4). Die Schritte 3 und 4 bezwecken, dass die Wahlbehörde keine gefälschten Stimmen abgeben kann. Nun hat der Wähler eine elektronische Wahlkarte bzw. Prüfkarte, die von der Registrierungsstelle der Behörde wie vom Trustcenter als gültig erklärt und blind unterschrieben wurde.

3. Die blinde Unterschrift basiert auf dem Verschlüsselungsverfahren von Rivet, Shamir und Adleman. Allerdings wendet der Unterschreibende seinen privaten Schlüssel auf das eigentliche Schriftstück und ein Beiwerk (Padding) an. Wird das Padding vom Empfänger der Unterschrift entfernt, bleibt das unterschriebene Schriftstück übrig, das nun anonym verwendet werden kann.

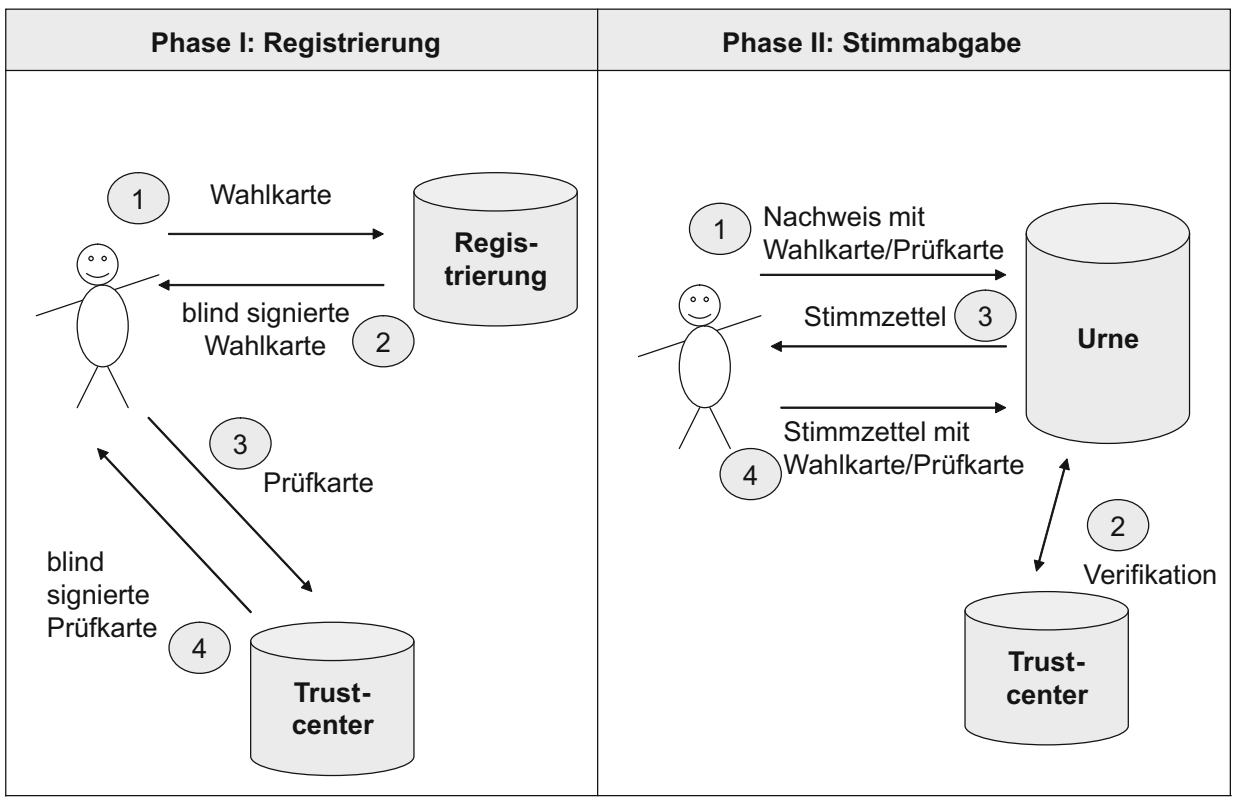

Abb. 2: Registrierung und Stimmabgabe (nach [Prosser \& Müller-Török 2002]) 
Die zweite Phase der Stimmabgabe in Abbildung 2 findet am Abstimmungs- oder Wahltag statt. Der Wähler verwendet dazu seine bereits verifizierte Wahl- bzw. Prüfkarte. Er schickt beide zum Nachweis an die Urne (Schritt 1), um einen gültigen Stimm- oder Wahlzettel zu erhalten. Bevor der Server der Urne den Stimmzettel retournieren kann, wird die Wahl- und Prüfkarte via Trustcenter verifiziert (Schritt 2). Läuft alles reibungslos ab, erhält der Wähler seine gewünschte Stimmkarte im Schritt 3. Er füllt seinen Stimm- oder Wahlzettel aus und schickt diesen mit seiner Wahlberechtigung (Wahlkarte/Prüfkarte) zurück an die Urne. Nach Ablauf der Wahl veröffentlicht der Server der Urne eine Liste der Stimmzettel und die zugehörigen elektronischen Wahlkarten.

\section{Analyse und Visualisierung mehrdimensionaler Wahlergebnisse}

In den Phasen eDiscussion und ePosting müssen die Informationen dargestellt und kommentiert werden, damit die Bürgerinnen und Bürger sich ein klareres Bild über Sachthemen oder politische Profile der Mandatsträger verschaffen können. Mit mehrdimensionalen Visualisierungstechniken ist es möglich, komplexe Sachzusammenhänge zu veranschaulichen. Beispielsweise werden am geografischen Institut der Universität Zürich Methoden der Kartografie benutzt [Hermann \& Leuthold 2000], um die Schweiz als Raum von Weltanschauungen darzustellen (Abb. 3).

Ausgangsbasis für die Raumdarstellung waren die Resultate aller eidgenössischen Volksabstimmungen in den Jahren 1981 bis 1999. Volksabstimmungen in der Schweiz sind Referenden zu Sachfragen aus der Politik, zu denen die Bürgerinnen und Bürger mit Ja oder Nein antworten können. Die Ja-Stimmenanteile von 3021 Gemeinden zu den Vorlagen wurden einer explorativen Faktoranalyse unterzogen. Die Faktoren bilden die Dimensionen des Raumes der Weltanschauungen, wobei eine zweidimensionale Ansicht dieses Raumes in der Abbildung 3 gegeben ist. Die im Raum lokalisierten politischen Ziele aus einer qualitativen Inhaltsanalyse werden durch Kreisscheibendiagramme dargestellt. Dabei entspricht die Diagrammfläche der Häufigkeit des Vorkommens des Ziels in den Abstimmungen. Farben (hier Grauwerte) zeigen die Zugehörigkeit der Ziele zu inhaltsanalytischen Kategorien.

Geeignet gewählte Projektionen mehrdimensionaler Räume oder weitere Methoden des Data Mining erlauben, komplexe politische Zusammenhänge zu veranschaulichen. Damit lassen sich politische Positionen und Verhaltensmuster von Regierungen, Parteien oder Interessenverbänden aufdecken. Zudem kann in Zeitabständen untersucht werden, ob Behördenmitglieder oder beauftragte Gremien den vom Volk, vom Parlament oder von der Regierung vorgegebenen Strategien folgen und die entsprechenden Programme umsetzen (Politcontrolling, vgl. Abschnitt 5).

Die diskutierten Visualisierungsmethoden dienen der Meinungsbildung und der Entscheidungsfindung im Rahmen einer eDemocracyPolitik. Beispielsweise können bei der Wahl politischer Abgeordneter für ein Parlament deren Profil aufgrund des politischen Handelns der vergangenen Legislaturperiode aufgezeigt werden (vgl. Abb. 4, in der Grafik als schwarz ausgezogenes Polygon dargestellt). Gruppen von Politikerinnen und Politikern, Fraktionen oder ganze Parteien können ebenfalls im Spinnennetz unterschiedlicher Zielsetzungen aufgrund ihres Verhaltens positioniert werden (gestricheltes Polygon in Abb. 4).

In der Schweiz werden politische Spinnenprofile im Vorfeld von Nationalratswahlen (Parlament) auf einer entsprechenden Webplattform und in den Medien publiziert [Jeitziner 2004]. Neben der Vorstellung der Kandidatinnen und Kandidaten, weiteren Angaben zur politischen Ausrichtung und dem Fähigkeitsprofil stellen die Spinnenprofile ein zusätzliches Beurteilungskriterium dar. Entsprechen- 


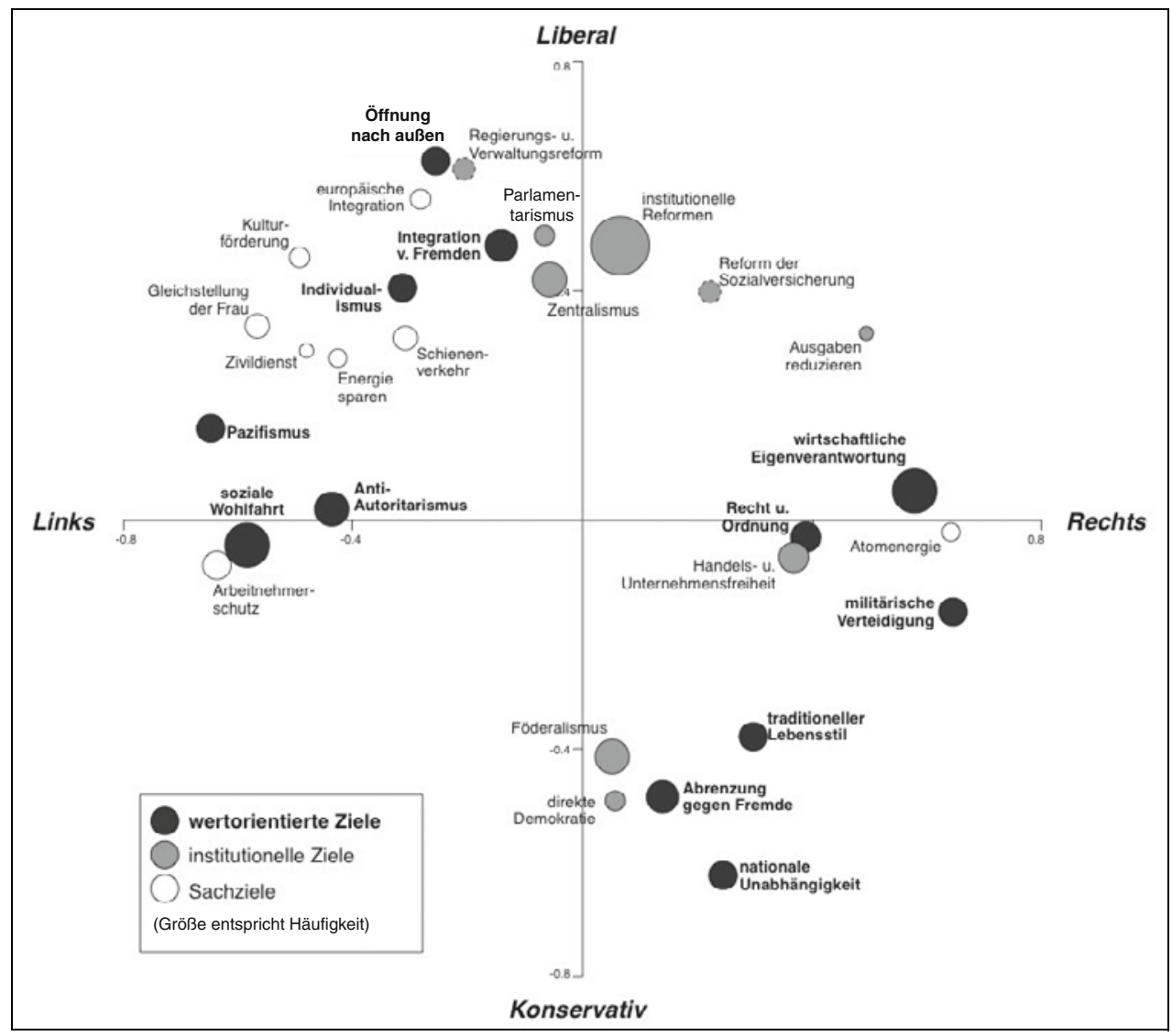

Abb. 3: Visualisierung der Lage und Häufigkeit politischer Ziele (nach [Hermann \& Leuthold 2000])

de Informationsportale zu Abstimmungen und Wahlen ergänzen die von den Parlamentariern selbst deklarierten Positionen und lassen erkennen, ob opportunistisch Programme angeboten werden, die primär auf die Wählerstimmung zugeschnitten sind, oder ob das persönliche Programm auch dem Verhalten des Parlamentariers entspricht.

Das Rating von Parlamentariern durch das systematische Auswerten des Abstimmungsverhaltens stellt eine Informationsverdichtung dar. Sie kann als eine mögliche Form eines politischen Controllings verstanden werden.

\section{Schritte zum Public Memory}

Der Historiker John Bodnar charakterisiert das Public Memory als "a body of beliefs and ideas about the past that help a public or society understand both its past, present, and by implication, its future " [Bodnar 1992]. Dieses öffentliche oder kulturelle Gedächtnis einer Gesellschaft kann durch die Digitalisierung wichtiger Werke, Dokumente, Bilder, Ansprachen, Filme, TV- und Radioaufzeichnungen, Regierungsprogramme und -beschlüsse, Bürgerinitiativen etc. in webbasierten Bibliotheken oder digitalen Archiven der Öffentlichkeit zur Verfügung gestellt werden. Dadurch erhalten 
nicht nur Juristen, Historiker oder weitere Experten Zugang zu den digitalisierten Originaldokumenten und Abbildungen, sondern auch alle Bewohnerinnen und Bewohner des Global Village.

Der Soziologe Niklas Luhmann, als ausgebildeter Jurist und Beamter in Lüneburg erfahren in den Verwaltungsabläufen, widmet in seinem Buch "Die Politik der Gesellschaft" [Luhmann 2000] dem Gedächtnis der Politik ein eigenes Kapitel. Er überträgt die von Neurobiologie und psychischen Systemen beeinflussten Gedächtnistheorien auf das kollektive Gedächtnis. Für politische Systeme ${ }^{4}$ und das politische Gedächtnis erklärt er "Werte« und »Interessen«

4. Luhmanns Systemtheorie basiert auf der Evolution von Kommunikation und auf der Evolution der Gesellschaft. als bedeutende Faktoren. Während subjektive Werte im politischen Gedächtnis eine moralische Instanz bilden, sind Interessen faktische Gegebenheiten jedes Bürgers. Das politische Gedächtnis steht im Spannungsfeld von Werten und Interessen und selektiert nach zu Vergessendem und Behaltendem. Luhmann sagt aus, dass jedes politische System mit der Unterscheidung von Werten und Interessen reagiert, nämlich "von Werten, die das anmahnende Erinnern ermöglichen und durch Legitimation der Desiderate ${ }^{5}$ unterstützen, und von Interessen, die, wenn sie sich Gehör verschaffen können und politisch wichtig sind, die kommunikative Aktualisierung besorgen«.

5. Ein Desiderat ist ein vermisstes und zur Anschaffung in Bibliotheken vorgeschlagenes Werk oder Dokument.

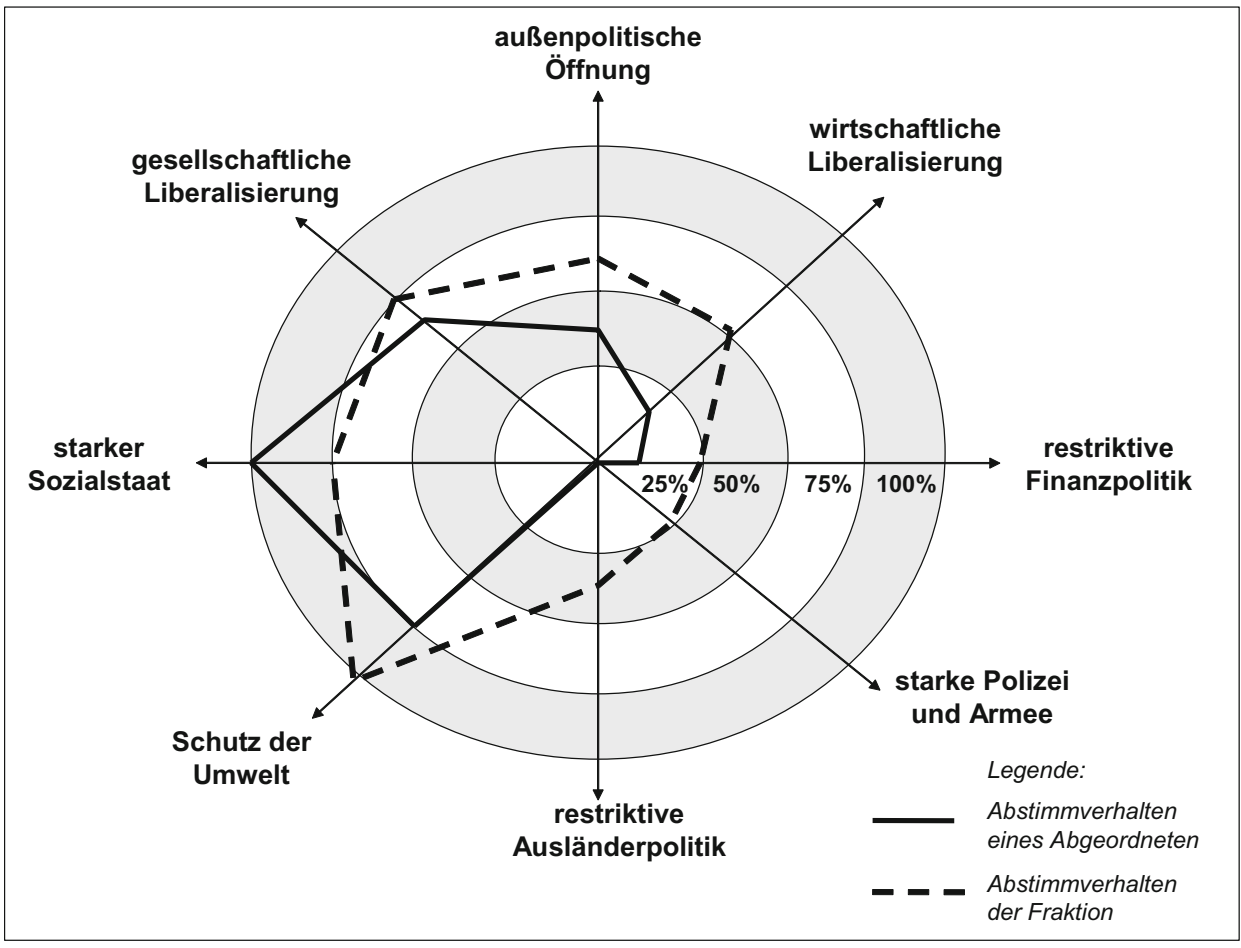

Abb. 4: Spinnenprofil eines Abgeordneten bzw. der zugehörigen Fraktion (angelehnt an die Forschungsarbeiten [Sotomo 2008] der Universität Zürich) 
Regierung und Verwaltung haben den Auftrag, neben der Veröffentlichung der Strategien, Programme und Aktivitäten und den dazu notwendigen Gesetzesvorlagen und Verordnungen auf dem eGovernement-Portal die vielfältigen Informationen zu publizieren und damit für Transparenz zu sorgen. Eine Informations- und Wissensgesellschaft ist ohne die Nutzung rechnergestützter Expertensysteme und Wissensbanken nicht überlebensfähig.

Wissensmanagement: Eine Gesellschaft kann mit geeigneten eGovernment-Programmen und Initiativen zu Public Memory den Zugang zu politisch relevantem Wissen verbessern. Die Weltbank beispielsweise beschäftigt mehrere Hundert Knowledge Worker, weil sie erkannt hat, dass dem Know-how und Wissenstransfer eine größere Bedeutung zukommt als dem Verleihen von Geld [WBI 2008].

Wirkungsorientierte Verwaltung: Regierungsstellen und Verwaltungseinheiten können Effizienz und Effektivität ihrer Aktivitäten steigern, wenn sie sich webbasierter Informations- und Workflow-Management-Systeme bedienen. Solche Systeme unterstützen virtuelle Organisations- und Zusammenarbeitsformen und erlauben, die Komplexität der öffentlichen Produkte und Dienstleistungen zu beherrschen.

Organisationales Wissen: Eine lernende Organisation oder Öffentlichkeit versucht, strukturelle Erkenntnisse und Know-how über Verwaltungs- und Beziehungsprozesse mit den Bürgerinnen und Bürgern als kollektives Gut zu erkennen und zu erhalten. Primär stehen hier nicht individuelle Bedürfnisse oder Werthaltungen im Vordergrund, sondern organisationale Entscheidungsverfahren und kollektive Erfahrungswerte.

Ein eGovernment-Portal muss für alle Bevölkerungsschichten und damit auch für Menschen mit körperlichen, geistigen und physischen Behinderungen zugänglich bleiben [WAI 2008]. Es bietet nicht nur die Behördendienste für Bürger und Unternehmen an, son- dern kann darüber hinaus auch Verfahren und Wissensbanken für eDemocracy umfassen. Der Weg zu einem Public Memory ist lang und steinig, da wichtige Themen wie Schutz der Privatsphäre der Bürgerinnen und Bürger, Urheberrechtsfragen bei digitalen Objekten, Archivierungskonzepte und -zeiten bei digitalen Speicherträgern etc. laufend geklärt und mit geeigneten Methoden und Techniken umgesetzt werden müssen. Nicht zuletzt ermöglicht ein Public Memory, realisiert in einem öffentlich zugänglichen eGovernment-Portal, ein demokratisches Politcontrolling und ebnet damit den Weg zur Informations- und Wissensgesellschaft.

\section{Literatur}

[Bodnar 1992] Bodnar, J.: Remaking America - Public Memory, Commemoration, and Patriotism in the Twentieth Century. Princeton University Press, 1992.

[Brandt \& Volkert 2002] Brandt, M.; Volkert, B.: E-Voting im Internet - Formen, Entwicklungsstand und Probleme. Arbeitsbericht Nr. 218 der Akademie für Technikfolgenabschätzung, Baden-Württemberg, 2002.

[Hermann \& Leuthold 2000] Hermann, M.; Leuthold, H.: Einsatz von kartographischen Methoden und GIS zur Analyse und Visualisierung mehrdimensionaler Strukturen in den Sozialwissenschaften. In: Schmidt, B.; Uhlenküken, C. (Hrsg.): Visualisierung Raumbezogener Daten Methoden und Anwendungen. Beiträge zum 3. GeoViSC-Workshop, Band II, Münster, 2000, S. 77-92.

[Jeitziner 2004] Jeitziner, B.: Wahlen im Internetzeitalter - Informationsvermittler als politische Berater von Wählern und Politikern. In: Schaltegger, C. A.; Schaltegger, S. C. (Hrsg.): Perspektiven der Wirtschaftspolitik. vdf Verlag, Zürich, 2004, S. 47-64.

[Krimmer 2006] Krimmer, R. (Hrsg.): Electronic Voting. Lecture Notes in Informatics. Volume P-86, 2006.

[Kurz \& Rieger 2007] Kurz, C.; Rieger, F.: NEDAPWahlcomputer - Manipulationsmethoden an Hard- und Software. Informatik-Spektrum, Band 30, Heft Nr. 5, Oktober 2007, S. 313-321. 
[Luhmann 2000] Luhmann, N.: Die Politik der Gesellschaft. Suhrkamp, Frankfurt, 2000.

[Meier 2002] Meier, A. (Hrsg.): E-Government. HMD - Praxis der Wirtschaftsinformatik, Heft 226, dpunkt.verlag, Heidelberg, 2002.

[Meier 2009] Meier, A.: eDemocracy \& eGovernment - Entwicklungsstufen einer demokratischen Wissensgesellschaft. Springer-Verlag, Heidelberg, 2009.

[Nurmi et al. 1991] Nurmi, H.; Salomaa, A.; Santean, L.: Secret Ballot Election in Computer Networks. Computers and Security, Vol. 36, No. 10, 1991, S. 553-560.

[Prosser \& Müller-Török 2002] Prosser, A.; MüllerTörök, R.: E-Democracy - Eine neue Qualität im demokratischen Entscheidungsprozess. Wirtschaftsinformatik, Vol. 44, Nr. 6, 2002, S. 545556.

[Prosser et al. 2002] Prosser, A.; Kofler, R.; Krimmer, R.; Unger, M. K.: e-Voting.at - Entwicklung eines Internet-basierten Wahlsystems für öffentliche Wahlen. Arbeitsbericht Nr. 4, Institut für Informationsverarbeitung und Informationswirtschaft, Wirtschaftsuniversität Wien, 2002.
[Sotomo 2008] Sotomo - Sociotopological Modeling, http://sotomo.geo.unizh.ch/research/; Zugriff am 22.07.2008.

[WAI 2008] Web Accessability Initiative (WAI): Web Content Accessability Guidelines (WCAG), 2008, www.w3.org/WAl/; Zugriff am 25.11.2008.

[WBI 2008] World Bank Institute (WBI): Knowledge Sharing, 2008, www.worldbank.org/ks/; Zugriff am 25.11.2008.
Prof. Dr. Andreas Meier Universität Fribourg Department of Informatics Boulevard de Pérolles 90 $\mathrm{CH}-1700$ Fribourg andreas.meier@unifr.ch http://diuf.unifr.ch/is/ 